Article

\title{
Comprehensive Analytical Approximations of the Pull-In Characteristics of an Electrostatically Actuated Nanobeam under the Influences of Intermolecular Forces
}

\author{
Hassen M. Ouakad * (1) and Jihad E. AlQasimi \\ Department of Mechanical Engineering, King Fahd University of Petroleum and Minerals, Dhahran 31261, \\ Saudi Arabia; jeqasimi@kfupm.edu.sa \\ * Correspondence: houakad@kfupm.edu.sa
}

Received: 14 December 2017; Accepted: 9 January 2018; Published: 12 January 2018

\begin{abstract}
In this paper, analytical closed-form expressions to accurately estimate the pull-in characteristics of an electrostatically-actuated doubly-clamped nanobeam are derived and examined. In this regard, a coupled electro-mechanical problem for the nano-actuator is first presented assuming a single mode approximation while taking into account all the possible structural, electrical and nanoscale effects: the fringing of the electrical actuating force, the geometric mid-plane stretching and intermolecular (van der Walls and Casimir) forces. The complicated nonlinear resultant equations are numerically approximated in order to derive the closed-form expressions for the important nano-actuator pull-in characteristics: i.e., the detachment length, the minimum reachable gap size before the collapse and the respective pull-in voltage. The resulting closed-form expressions are first quantitatively validated with other previously published results, and comparisons showed an acceptable agreement. Unlike the reported expressions in the literature, the proposed closed-form expressions in this work are proper approximations, fairly accurate and, more importantly, provide a quick estimate of the critical design pull-in parameters of the nano-actuator. In addition, the analysis of these expressions demonstrated that the consideration of the intermolecular forces together with the fringe effect tends to significantly reduce the threshold pull-in voltage, whereas the mid-plane stretching parameter tends to the contrary to increase the voltage at the pull-in collapse. The derived expressions of these analytical/approximate solutions could hopefully be appropriately used by NEMS engineers as simple/quick procedures for successful design and fabrication of electrostatically-actuated nano-devices.
\end{abstract}

Keywords: NEMS; actuator; analytical solution; pull-in characteristics; mid-plane stretching; van der Waals force; Casimir force

\section{Introduction}

Recently, the field of Nanoelectromechanical Systems (NEMS) has become one of the most emerging fundamental and applied research areas. It is essentially considered as a multi-disciplinary research branch involving many engineering applications such as: nano-switches, nano-tweezers, nano-grippers, nano-resonators, nano-actuators, etc. [1]. Estimating accurately the pull-in characteristics of NEMS-based devices has been the center of research attention over the past few years, mostly because predicting these parameters forms the basis of utilizing these tiny structures as sensors and actuators of distinguishing properties. In addition, these characteristics could represent, for the majority of the designers in this area, an effective way to extract the mechanical properties of such nano-structures.

One of the most assumed configurations in designing NEMS devices is the clamped-clamped nanobeam arrangement. Clamped-clamped NEMS-based nanobeams have been under extensive 
research in the NEMS community, and they find potential in many applications such as random access memory, resonating devices for high-frequency operation, fast switching in communication networking applications [2], energy harvesters [3], etc.

Among the different actuation mechanisms for such nano-structures, the electrostatic actuation technique is the most commonly used because of its numerous advantages such as: the large force generation, the easy Complementary Metal-Oxide-Semiconductor (CMOS) integration, the compatibility with all the micro-/nano-manufacturing processes, etc. However, it is worth noting that the efficiency of this technique is restricted by the well-known structural instability named the "pull-in" collapse [4]. This structural instability was first discovered for micro-actuators and revealed to be a function of the actuating electric force [4]. However, going down further in the nano scale, this structural instability was shown to be dependent on other forces, mainly the intermolecular forces, for nanobeam-based actuators. It should be pointed out that the nano-actuator where the initial gaps are below $100 \mathrm{~nm}$, the intermolecular forces (Casimir and van der Waals forces essentially) are considered prominent and strong and become comparable to the electrostatic actuating forces and even overriding other attractive intermolecular forces. During the pull-in instability scenario, the nanobeam restoring force (i.e., its overall stiffness) is no longer capable of balancing the electric actuating force, resulting in the collapse of the nano-structure. Furthermore, other than the nano-structure restoring and actuating forces, other highly nonlinear intermolecular forces such as the van der Waals and the Casimir forces may even significantly distress the pull-in characteristics of the nano-actuator. Occasionally, these intermolecular forces may lead to the nano-structure restoring elastic forces depending on the initial gap size and therefore may lead to collapse even without the need to consider any electric actuating voltage $[5,6]$.

An extra and important non-linear effect in doubly-clamped nanobeam designs is the geometric mid-plane stretching effect. This effect frequently results in a significant increase in beam stiffness (i.e., its resulting restoring elastic force) and consequently may drastically alter its pull-in characteristics. Considering the combined effects of the intermolecular forces and the mid-plane stretching effect is extremely important for the successful design, analysis and fabrication of NEMS-based electrostatically-actuated nanobeams. Though several extensive investigations on the pull-in characteristics of NEMS devices, mainly assuming semi-analytical, analytical and closed-form approximations for different nano-cantilever configurations [5-9], are available in recent literature, only a few attempts [10-15] performed a similar analysis on the doubly-clamped nanobeam while taking into consideration the combined effects of the intermolecular forces and the nonlinear mid-plane stretching effect. Most of the cited literature investigated the doubly-clamped nanobeam pull-in characteristics assuming a lumped system modeling and/or numerical simulation approaches $[10,11]$. Others included in their modeling the nonlocal elasticity theory [12], the modified strain gradient theory [13], the modified Adomian method [14], the hybrid nonlocal beam model [15], etc. Only recently, Shokravi [16] investigated the dynamic pull-in and pull-out problems of nanoplate-based NEMS switches under electrostatic and Casimir forces assuming Eringen's nonlocal theory [16].

Another major effect at the nano-scale that may alter nanobeam-based actuators is their surface effects. Few previous experiments [17] demonstrated that the surface layer plays an important role in the structural behavior of nanoscale-based structures. In the classical continuum mechanics, the effect of the surface layer is typically ignored. However, for nanoscale devices, because of high surface-to-volume ratios, the influence of the surface layer on the overall dynamic behavior of the nanostructure could no longer be neglected $[18,19]$. Therefore, in one of our previous investigations [20], we studied the instability characteristics of free-standing nanowires based on the consideration of Casimir attractive forces in addition to the surface effects.

The accessibility to simple analytical expressions / generalized closed-form solutions for estimating the pull-in characteristics of doubly-clamped NEMS-based nanobeams is still not properly investigated in the literature. Although few groups $[12,13]$ attempted to formulate analytical solutions for the pull-in instability of nano-devices, their methods mostly suffer from complexity (complicated mathematical 
operations) and numerical integration techniques. Another limitation is that the final closed-form expression involves and also is dependent on the 'pull-in deflection' factor [12,13], which itself requires further computation.

Therefore, the present work aims at deriving simple generalized closed-form expressions for estimating the critical pull-in design characteristics of the fixed-fixed nanobeam that hopefully will serve as fast, simple and accurate design guidelines for NEMS engineers. The plan is first to formulate the doubly-clamped nanobeam problem assuming a Euler-Bernoulli beam model, while considering the mid-plane stretching effect, the van der Waals and Casimir forces and the nonlinear quadratic electrostatic forces while accounting for the fringing effects. Then, a Galerkin-based modal decomposition technique will be carried out. Its resulting integrals and higher-order polynomials will be solved numerically to acquire the doubly-clamped nanobeam pull-in parameters (the critical dimension limits, the maximum detachment length and the minimum gap size to pull-in). Afterward, a curve fitting process will be used to provide simple and readymade analytical expressions for the pull-in voltage function of the mid-plane stretching, the fringing electric field and the intermolecular force parameters.

The main advantage of the above planned approach is that the derived analytical expressions are independent of the nanobeam deflection unlike the expressions previously reported in the literature. In addition, the expressions will not include complicated integrations, nor even difficult mathematical operations, hence reducing the resulting computation cost. All these derived expressions will then be compared and validated quantitatively with the results from available literature. The incorporation of the mid-plane stretching effect, the intermolecular forces and fringing effect of the electric actuating field can significantly affect the overall pull-in characteristic of such a nano-actuator. Accordingly, the paper is organized as follows: Section 2 discusses the detailed analytical/numerical modeling of the electrically-actuated nanobeam taking into account the nonlinear mid-plane stretching effect, the intermolecular effects and the fringing field effect of the actuating electric force. Then, the numerical results and discussions of the proposed analytical expressions and their respective validation with reported literature are summarized in Section 3. Finally, the main contributions and conclusions are provided in Section 4.

\section{Analytical Modeling}

We consider here an isotropic clamped-clamped nanobeam of length $L$, a uniform rectangular cross-section area of $A=b h$ where $b$ is the beam width, $h$ its respective thickness, an effective Young's modulus $E$, an area moment of inertia $I=\left(b h^{3}\right) / 12$ and with an initial gap size between the flexible beam and its respective grounded actuating electrode of $d$, as shown in Figure 1. The governing differential static equation of motion of the nanobeam resulting bending deflection $w(x)$ under the influence of the electrostatic, as well as the intermolecular force can be written as follows [10]:

$$
E I \frac{d^{4} w}{d x^{4}}=F_{\text {electrical }}(w)+F_{\text {stretching }}(w)+F_{\text {Intermolecular }}(w)
$$

where $F_{\text {electrical }}$ symbolizes the actuating electrostatic force per unit length including the first order fringing fields effect, $F_{\text {stretching }}$ represents the mid-plane stretching effect and the $F_{\text {Intermolecular }}$ term denotes the intermolecular forces. All these forces can be written respectively as follows [10,12]:

$$
\begin{gathered}
F_{\text {electrical }}(w)=\frac{\varepsilon b V_{D C}^{2}}{2(d-w)^{2}}\left(1+0.65 \frac{(d-w)}{b}\right) ; F_{\text {stretching }}(w)=\frac{E A}{2 L}\left[\int_{0}^{l}\left(\frac{d w}{d x}\right)^{2} d x\right] \frac{d^{2} w}{d x^{2}} \\
F_{\text {Intermolecular }}(w)=\underbrace{\frac{H b}{6 \pi(d-w)^{3}}}_{=F_{v d W}(w)}+\underbrace{\frac{\pi^{2} h c b}{240(d-w)^{4}}}_{=F_{\text {Casimir }}(w)}
\end{gathered}
$$


where $\varepsilon=8.854 \times 10^{-12} \mathrm{~F} / \mathrm{m}$ is the air permittivity, $V_{D C}$ is the applied actuating $D C$ voltage across the nanobeam and its stationary substrate, $H$ is the Hamaker constant defined for a van der Waals (vdW) body-body interaction with values in the range of $10^{-19} \mathrm{~J}[21], c=3 \times 10^{8} \mathrm{~m} / \mathrm{s}$ is the speed of light and $\hbar=1.055 \times 10^{-34} \mathrm{~J}^{-\mathrm{s}}$ is Planck's constant.

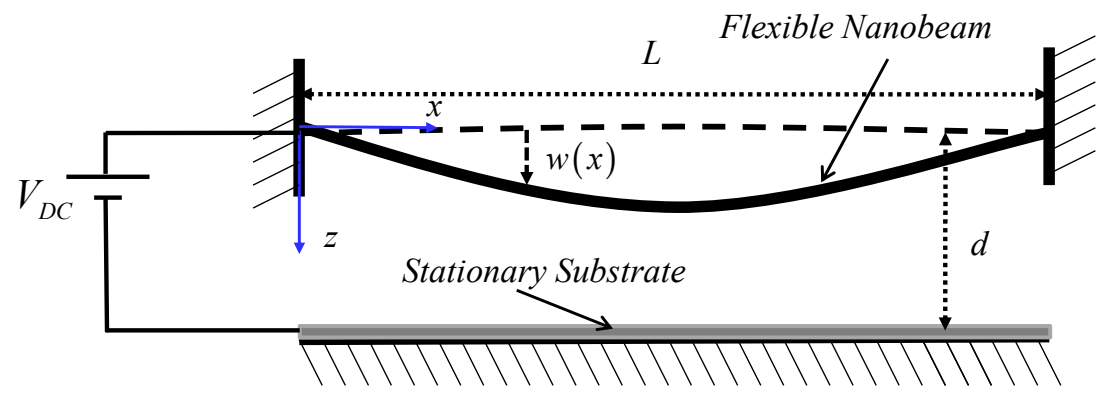

Figure 1. Schematic of an electrostatically-actuated fixed-fixed nanobeam.

In the below, we will consider two cases: one with only the van der Waals force; and the second case will treat the nanobeam under the Casmir force.

\subsection{First Case: van der Waals Force Only $\left(F_{v d W} \neq 0\right.$ and $\left.F_{\text {Casimir }}=0\right)$}

In this case, we assume that the intermolecular forces are the van der Waals force only. Therefore, Equation (1) can be written as [22,23]:

$$
E I \frac{d^{4} w}{d x^{4}}=\frac{\varepsilon b V_{D C}^{2}}{2(d-w)^{2}}+\frac{0.65 \varepsilon V_{D C}^{2}}{2(d-w)}+\frac{E A}{2 L}\left[\int_{0}^{L}\left(\frac{d w}{d x}\right)^{2} d x\right] \frac{d^{2} w}{d x^{2}}+\frac{A b}{6 \pi(d-w)^{3}}
$$

Next and for convenience, we introduce the following non-dimensional parameters ( $\hat{w}=w / d$ and $\hat{x}=x / L$ ) to normalize the above equation of motion. Hence, and after dropping the hats, Equation (3) can be expressed in a nondimensionalized form as follows [22,23]:

$$
\frac{d^{4} w}{d x^{4}}=\alpha_{e} V_{D C}^{2}\left[\frac{1}{(1-w)^{2}}+\frac{0.65 \alpha_{f f}}{(1-w)}\right]+\alpha_{s t}\left[\int_{0}^{1}\left(\frac{d w}{d x}\right)^{2} d x\right] \frac{d^{2} w}{d x^{2}}+\frac{\alpha_{v d W}}{(1-w)^{3}}
$$

where the above introduced nondimensional coefficients are given as follows:

$$
\alpha_{e}=\frac{\varepsilon b L^{4}}{2 E I d^{3}} ; \alpha_{f f}=\frac{d}{b} ; \alpha_{s t}=6\left(\frac{d}{h}\right)^{2} ; \alpha_{v d W}=\frac{2 H L^{4}}{\pi E h^{3} d^{4}} ;
$$

Next, Equation (4) can be rewritten as [23]:

$$
(1-w)^{3} \frac{d^{4} w}{d x^{4}}=\alpha_{e} V_{D C}^{2}\left[(1-w)+0.65 \alpha_{f f}(1-w)^{2}\right]+\alpha_{s t}(1-w)^{3}\left[\int_{0}^{L}\left(\frac{d w}{d x}\right)^{2} d x\right] \frac{d^{2} w}{d x^{2}}+\alpha_{v d W}
$$

Numerous methods can be used to numerically solve the above nonlinear equation: such as the finite-element method [24], the finite-difference method [25], the shooting method [26,27], the differential-quadrature method [27], etc., which are all considered to be computationally expensive and in some cases unstable since some rely on initial guesses. In this investigation, we propose to use Galerkin-based reduced-order modeling that transforms the above nonlinear governing differential equation into a nonlinear algebraic equation system (considering only static DC load). Assuming now 
the Galerkin-based modal discretization technique [28,29], the nanobeam deflection solution of the above Equation (6) can be written as follows:

$$
w(x)=\sum_{i=1}^{N} k_{i} \Psi_{i}(x)
$$

where $N$ is the assumed number of modes in the discretization method, $k_{i}$ are unknown constant coefficients and $\Psi_{i}(x)$ denote the beam modal functions satisfying the doubly-clamped boundary conditions at $x=0$ and $x=1$. Substituting Equation (7) into Equation (6), we get the following discretized equation:

$$
\begin{aligned}
\sum_{i=1}^{n}\left(1-\sum_{i=1}^{n} k_{i} \Psi_{i}(x)\right)^{3} k_{i} \Psi_{i}^{\prime \prime \prime \prime}(x) & =\alpha_{e} V_{D C}^{2}\left[\left(1-\sum_{i=1}^{n} k_{i} \Psi_{i}(x)\right)+0.65 \alpha_{f f}\left(1-\sum_{i=1}^{n} k_{i} \Psi_{i}(x)\right)^{2}\right]+ \\
& +\alpha_{s t}\left(1-\sum_{i=1}^{n} k_{i} \Psi_{i}(x)\right)^{3}\left[\int_{0}^{1}\left(\sum_{i=1}^{n} k_{i} \Psi_{i}^{\prime}(x)\right)^{2} d x\right] \sum_{i=1}^{n} k_{i} \Psi_{i}^{\prime \prime}(x)+\alpha_{v d W}
\end{aligned}
$$

Subsequently, multiplying both sides of Equation (8) with a modal function $\Psi_{1 \leq j \leq n}(x)$ and integrating with respect to $x$ in $0-1$, we obtain:

$$
\begin{aligned}
\int_{0}^{L} \Psi_{j}\left(\sum_{i=1}^{N}\left(1-\sum_{i=1}^{N} k_{i} \Psi_{i}\right)^{3} k_{i} \Psi_{i}^{\prime \prime \prime \prime}\right) d x & =\alpha_{e} V_{D C}^{2}\left[\int_{0}^{L} \Psi_{j}\left(\left(1-\sum_{i=1}^{N} k_{i} \Psi_{i}\right)+0.65 \alpha_{f f}\left(1-\sum_{i=1}^{N} k_{i} \Psi_{i}\right)^{2}\right) d x\right]+ \\
& +\alpha_{s t}\left[\int_{0}^{1}\left(\sum_{i=1}^{N} k_{i} \Psi_{i}^{\prime}\right)^{2} d x\right] \int_{0}^{L} \Psi_{j}\left(\left(1-\sum_{i=1}^{N} k_{i} \Psi_{i}\right)^{3} \sum_{i=1}^{N} k_{i} \Psi_{i}^{\prime \prime}\right) d x+\alpha_{v d W} \int_{0}^{L} \Psi_{j} d x
\end{aligned}
$$

Based on the below normality conditions [30] of the modal functions $\Psi_{1 \leq j \leq n}(x)$,

$$
\int_{0}^{1} \Psi_{i}(x) \Psi_{j}(x) d x=\left\{\begin{array}{lll}
0 & \text { if } & i \neq j \\
1 & \text { if } & i=j
\end{array} ; \text { and } \Psi_{i}^{\prime \prime \prime \prime}(x)=\omega_{i}^{2} \Psi_{i}(x)\right.
$$

Equation (9) can be re-written as:

$$
\begin{aligned}
\sum_{i=1}^{N} \int_{0}^{1} \Psi_{j} \omega_{i}^{2} k_{i} \Psi_{i}\left(1-\sum_{i=1}^{N} k_{i} \Psi_{i}\right)^{3} d x & =\alpha_{e} V_{D C}^{2}\left[\int_{0}^{1} \Psi_{j}\left(1-\sum_{i=1}^{N} k_{i} \Psi_{i}\right) d x+0.65 \alpha_{f f} \int_{0}^{1} \Psi_{j}\left(1-\sum_{i=1}^{N} k_{i} \Psi_{i}\right)^{2} d x\right]+ \\
& +\alpha_{s t}\left[\int_{0}^{1}\left(\sum_{i=1}^{N} k_{i} \Psi_{i}^{\prime}\right)^{2} d x\right] \int_{0}^{1} \Psi_{j}\left(\left(1-\sum_{i=1}^{N} k_{i} \Psi_{i}\right)^{3} \sum_{i=1}^{N} k_{i} \Psi_{i}^{\prime \prime}\right) d x+\alpha_{\nu d W} \int_{0}^{1} \Psi_{j} d x
\end{aligned}
$$

Next and assuming only one mode approximation $(N=1)$, Equation (11) can be simplified to the following form:

$$
\begin{aligned}
\omega_{1}^{2} k_{1} \int_{0}^{1} \Psi_{1}^{2}\left(1-k_{1} \Psi_{1}\right)^{3} d x & =\alpha_{e} V_{D C}^{2}\left[\int_{0}^{1} \Psi_{1}\left(1-k_{1} \Psi_{1}\right) d x+0.65 \alpha_{f f} \int_{0}^{1} \Psi_{1}\left(1-k_{1} \Psi_{1}\right)^{2} d x\right]+ \\
& +\alpha_{S t} \int_{0}^{1} \Psi_{1}\left(1-k_{1} \Psi_{1}\right)^{3}\left[\int_{0}^{1}\left(k_{1} \Psi_{1}^{\prime}\right)^{2} d x\right] k_{1} \Psi_{1}^{\prime \prime} d x+\alpha_{v d W} \int_{0}^{1} \Psi_{1} d x
\end{aligned}
$$

The normalized first modal frequency and its corresponding normalized mode shape function considering a doubly-clamped beam configuration are given respectively as [30]: 


$$
\left\{\begin{array}{l}
\omega_{1} \approx 22.3733 \\
\Psi_{1}(x) \approx \cosh \left(\sqrt{\omega_{1}} x\right)-\cos \left(\sqrt{\omega_{1}} x\right)-0.9825 \sinh \left(\sqrt{\omega_{1}} x\right)+0.9825 \sin \left(\sqrt{\omega_{1}} x\right)
\end{array}\right.
$$

Computing numerically all the integrations in Equation (12), we get the following nonlinear algebraic equation function of the unknown constant coefficient $k_{1}$ :

$$
\begin{aligned}
& 511.43 \alpha_{s t} k_{1}^{6}-1041.26 \alpha_{s t} k_{1}^{5}+\left(1327.05+706.417 \alpha_{s t}\right) k_{1}^{4}-\left(2781+151.35 \alpha_{s t}\right) k_{1}^{3}+ \\
& +\left(1996.32+2.0452 \alpha_{f f} \alpha_{e} V_{D C}^{2}\right) k_{1}^{2}-\left(500.56+\alpha_{e} V_{D C}^{2}+1.35 \alpha_{f f} \alpha_{e} V_{D C}^{2}\right) k_{1}+ \\
& +0.83087\left(\alpha_{e} V_{D C}^{2}+0.65 \alpha_{f f} \alpha_{e} V_{D C}^{2}+\alpha_{v d W}\right)=0
\end{aligned}
$$

\subsection{Second Case: Casimir Force Only $\left(F_{v d W}=0\right.$ and $\left.F_{\text {Casimir }} \neq 0\right)$}

In this sub-section, we only consider the intermolecular effect of the Casimir force; therefore, Equations (1) and (2) can be written in normalized form as follows:

$$
\frac{d^{4} w}{d x^{4}}=\alpha_{e} V_{D C}^{2}\left[\frac{1}{(1-w)^{2}}+\frac{0.65 \alpha_{f f}}{(1-w)}\right]+\alpha_{s t}\left[\int_{0}^{1}\left(\frac{d w}{d x}\right)^{2} d x\right] \frac{d^{2} w}{d x^{2}}+\frac{\alpha_{c a s}}{(1-w)^{4}}
$$

where, the nondimensional coefficient $\alpha_{c a s}$ is equal to $\frac{\pi^{2} h c L^{4}}{20 E h^{3} d^{5}}$.

Next, and following the same steps (Equations (6) to (13)) as in the first case, assuming only one mode in Galerkin's discretization technique and integrating numerically all the integrals, we get the following nonlinear algebraic equation function of the unknown constant coefficient $k_{1}$ :

$$
\begin{aligned}
& 758.49 \alpha_{s t} k_{1}^{7}-2045 \alpha_{s t} k_{1}^{6}+\left(1934+2082.52 \alpha_{s t}\right) k_{1}^{5}-\left(5308.19+941.89 \alpha_{s t}\right) k_{1}^{4} \\
& +\left(5561.97+151.35 \alpha_{s t}+1.2038 \alpha_{f f} \alpha_{e} V_{D C}^{2}\right) k_{1}^{3}-\left(2664.77+1.3294 \alpha_{e} V_{D C}^{2}+2.5923 \alpha_{f f} \alpha_{e} V_{D C}^{2}\right) k_{1}^{2}+ \\
& +\left(500.56+2 \alpha_{e} V_{D C}^{2}+4.615 \alpha_{f f} \alpha_{e} V_{D C}^{2}\right) k_{1}-0.83087\left(\alpha_{e} V_{D C}^{2}+0.65 \alpha_{f f} \alpha_{e} V_{D C}^{2}+\alpha_{c a s}\right)=0
\end{aligned}
$$

The derived two algebraic equations for the case of van der Waals attraction as the only intermolecular force, Equation (14), and for the case of Casimir force, Equation (16), are both nonlinear functions of the unknown coefficient $k_{1}$. In addition both equations are related to the electric force coefficient $\left(\alpha_{e}\right)$, the fringing fields effect $\left(\alpha_{f f}\right)$, the mid-plane stretching parameter $\left(\alpha_{s t}\right)$ and the intermolecular forces ( $\alpha_{v d W}$ for the first case and $\alpha_{c a s}$ for the second case). Therefore, these equations will be subsequently used in the following section to compute the nanobeam pull-in design parameters, as well as to derive general closed-form expressions for the pull-in parameters under the influence of the considered intermolecular forces. Finally, it is worth mentioning that because the above expressions, Equations (14) and (16), are respectively the sixth and seventh order polynomial function of $P_{1}$, only one solution in both cases has been found to be real and, hence, as the unique physical solution of the equilibrium deflection of the nanobeam.

\section{Results and Discussion}

In this section, the effects of each individual intermolecular force (i.e., the van der Waals and Casimir attraction forces, respectively) on the nanobeam deflection and pull-in instability are examined, and then general closed-form expressions for the maximum nanobeam detaching length and its respective minimum gap spacing are derived assuming zero DC load. Then, the effects of the intermolecular forces and mid-plane stretching parameters on the critical pull-in parameters are considered, and the corresponding closed-form pull-in expressions are presented. Finally, all the derived closed-form expressions are to be compared and validated with the other published results from the recent literature. 
3.1. Closed-Form Solutions for the Detachment Length and Its Respective Minimum Gap Size for an Un-Actuated Nanobeam

As mentioned earlier in the introductory part of this work, in the case of the nanobeam with a small gap size, the intermolecular forces become significantly dominant over the elastic restoring forces of the nano-actuator, resulting in an earlier stiction (collapse) even without assuming any electrical actuating potential yet. Hence, it is imperative for NEMS designers to be aware of these critical dimensions as a function of the assumed maximum designed nanobeam length and minimum actuator gap size of the freestanding nanobeam-based NEMS device.

Let us first consider the effect of van der Waals forces only and with zero DC load (i.e., $\alpha_{e} V_{D C}^{2}=0$ and $\alpha_{s t}=0$ in Equation (14)). The resulting polynomial expression is numerically solved for various values of the van der Waal parameter $\alpha_{v d W}$. The parameter $\alpha_{v d W}$ is assumed to vary from $0-50$, and the corresponding real root (physical solution) of the polynomial expression is computed while iterating the $\alpha_{v d W}$ parameter. The iteration process is carried out until a critical value of $\alpha_{v d W}$, denoted by $\alpha_{v d W}^{c r}$, starting from which the physical deflection of the nanobeam becomes an imaginary root, is achieved (as $\alpha_{v d W}^{c r} \approx 48.6$ ). The corresponding critical maximum nanobeam deflection is calculated to be equal to $\approx 0.309$. Therefore, $\alpha_{v d W}^{c r}=\frac{2 H L_{c r}^{4}}{\pi E h^{3} d_{c r}^{4}} \approx 48.6$, from which we can derive the expression of the maximum possible nanobeam detachment length and its respective minimum gap size respectively as follows:

$$
\left\{\begin{array}{l}
L_{c r}=\sqrt[4]{\frac{48.6 \pi E h^{3} d^{4}}{2 H}} \\
d_{c r}=\sqrt[4]{\frac{2 H L^{4}}{\alpha_{v d W}^{c r} \pi E h^{3}}}
\end{array} ;\right.
$$

Next and similarly as was done above for the case of the van der Waals attractive force, let us now consider the effect of the Casimir force only while neglecting both the electrostatic DC and the nonlinear mid-plane stretching effect (i.e., $\alpha_{e} V_{D C}^{2}=0$ and $\alpha_{s t}=0$ in Equation (16)). The resulting polynomial expression is then mathematically solved assuming various values of the Casimir force parameter $\alpha_{\text {cas }}$. This normalized parameter is iteratively varied from $0-50$, and the unique real root (the physical solution) of the nonlinear equation is calculated. Accordingly, a critical value $\alpha_{c a s}^{c r}$ from which the calculated physical solution becomes imaginary was found to be equal to $\approx 37.86$. Its corresponding nanobeam critical maximum deflection is obtained to be equal to $\approx 0.249$. Then, we can compute from Equation (15) that $\alpha_{c a s}^{c r}=\frac{\pi^{2} h c L_{c r} 4}{20 E h^{3} d_{c r}^{5}} \approx 37.86$, from which we can derive analytical expressions of both the maximum possible nanobeam detachment length and its respective minimum gap size respectively as follows:

$$
\left\{\begin{array}{l}
L_{c r}=\sqrt[4]{\frac{757 E h^{3} d^{5}}{\pi^{2} h c}} \\
d_{c r}=\sqrt[5]{\frac{\pi^{2} h c l^{4}}{757 E h^{3}}}
\end{array}\right.
$$

The above computed analytical expressions, Equations (17) and (18), can be used to properly design appropriate guidelines for the corresponding maximum length for a given gap size and correspondingly a suitable minimum gap size for a given length for a doubly-clamped nanobeam-based NEMS device.

\subsection{Comparison with the Literature Assuming an Un-Actuated Nanobeam}

In the below, the above computed analytical expressions will be compared with other previously published results for validation purposes. Tables 1 and 2 summarize the comparison of the calculated critical values for the normalized van der Waals parameter $\left(\alpha_{v d W}^{c r}\right)$ and Casimir parameter $\left(\alpha_{c a s}^{c r}\right)$, respectively, and their corresponding maximum reachable nanobeam deflections with other available results in the literature $[11,12,14]$. Table 1 clearly shows that the critical value of the $\alpha_{v d W}$ parameter 
obtained using the present work does not agree well with the results of [11,12]. Nonetheless, Table 2 indicates that the critical value of the $\alpha_{c a s}$ parameter agrees with the cited reference [14], but with a minor discrepancy that can be attributed to the single mode approximation assumed in the above analysis. However, the proposed methodology is purely analytical and provides simpler expressions in predicting properly and quickly the threshold limits for the cited basic design parameters and therefore better and successful design with no possibility of damage/collapse of these nanobeams during their fabrication process.

Table 1. Comparison of the critical van der Waal parameter using Equation (14) and its corresponding maximum nanobeam deflection with the results of [11,12].

\begin{tabular}{cccc}
\hline Parameter & Equation (14) & Results of [11] & Results of [12] \\
\hline$\alpha_{v d W}^{c r}$ & 0.3095 & - & 0.250 \\
$w_{\max } @ \alpha_{v d W}^{c r}($ in nm) & 48.625 & 40.4 & 57.857 \\
\hline
\end{tabular}

Table 2. Comparison of the critical Casimir parameter using Equation (16) and its corresponding maximum nanobeam deflection with the results of [14].

\begin{tabular}{ccc}
\hline Parameter & Equation (16) & Results of [14] \\
\hline$\alpha_{\text {cas }}^{c r}$ & 0.249 & 0.230 \\
$w_{\max } @ \alpha_{\text {cas }}^{c r}($ in nm) & 37.865 & 39.310 \\
\hline
\end{tabular}

Afterward, an additional comparison is carried out in Table 3. In the table is the comparison of the outcomes of both Equations (17) and (18) in calculating both the maximum possible nanobeam detachment length and its respective minimum gap size respectively with the reported results of [15]. A doubly-clamped nanobeam with modulus of elasticity $E=176 \mathrm{GPa}$, cross-sectional area $A=2.96 \times 10^{-19} \mathrm{~nm}^{2}$, thickness $h=3.5 \mathrm{~nm}$ and width $b=18 \mathrm{~nm}$ is assumed.

Table 3. Comparison of the threshold nanobeam detachment length $L_{c r}$ and its respective minimum gap size $d_{c r}$ obtained using Equation (17) and (18) for the case of van der Waals force and Casimir force, respectively, with the results of [15].

\begin{tabular}{|c|c|c|c|c|}
\hline \multirow{2}{*}{ Parameter } & \multicolumn{2}{|c|}{ Van der Waals Force Case } & \multicolumn{2}{|c|}{ Casimir Force Case } \\
\hline & Equation (17) & Results of [15] & Equation (18) & Results of [15] \\
\hline \multirow{2}{*}{$L_{c r}($ in $n m)$} & \multicolumn{2}{|c|}{$d=16 \mathrm{~nm}$} & \multicolumn{2}{|c|}{$d=25 \mathrm{~nm}$} \\
\hline & 298.85 & 312 & 324.77 & 340 \\
\hline \multirow{2}{*}{$d_{c r}($ in $\mathrm{nm})$} & \multicolumn{4}{|c|}{$L=200 \mathrm{~nm}$} \\
\hline & 5.31 & 5.1 & 9.73 & 9.5 \\
\hline
\end{tabular}

In the last part of this comparison sub-section, we propose to add the mid-plane stretching effect and compare again the calculated critical values of the van der Waals $\alpha_{v d W}^{c r}$ and Casimir $\alpha_{c a s}^{c r}$ parameters for un-actuated doubly-clamped nanobeams, using Equations (14) and (16) correspondingly. It is clearly demonstrated in Table 4 that as the mid-plane stretching parameter $\alpha_{s t}$ increases, the values of both critical parameters $\alpha_{v d W}^{c r}$ and $\alpha_{c a s}^{c r}$ also increase considerably. The reason behind this is that the mid-plane stretching increases the overall stiffness of the nanobeam (i.e., its resulting restoring force) that in turn makes the nanostructure harder (hardening behavior); hence, it can withstand higher intermolecular forces before collapsing. The values obtained through the present analytical/numerical approach are in acceptable agreement with the reported numerical, but non-closed-form solutions of [10], as can be perceived in Table 4. 
Table 4. Effect of the mid-plane stretching parameter on the critical intermolecular force parameters as compared with the results of [10].

\begin{tabular}{ccccc}
\hline \multirow{2}{*}{$\boldsymbol{\alpha}_{s t}$} & \multicolumn{2}{c}{$\boldsymbol{\alpha}_{v d W}^{c r}$} & \multicolumn{2}{c}{$\boldsymbol{\alpha}_{\text {cas }}^{\text {cr }}$} \\
\cline { 2 - 5 } & Equation (14) & Results of [10] & Equation (16) & Results of [10] \\
\hline 6 & 51.5 & 53.5 & 39.3 & 40.89 \\
12 & 54.5 & 57.7 & 41.1 & 42.29 \\
18 & 57.7 & 62.57 & 42.3 & 44.62 \\
24 & 61.1 & 67.73 & 43.9 & 47.29 \\
\hline
\end{tabular}

\subsection{Closed-Form Solutions for the Critical Pull-In Voltage for an Actuated Nanobeam}

So far, we have considered only the case of an un-actuated nanobeam (zero DC electrostatic load), and we subsequently investigated the nanobeam collapse problem governed by only the intermolecular van der Waals and Casimir forces. In this sub-section, the effect of the applied DC load will be investigated. Equations (14) and (16) are used to obtain the closed-form expressions for the pull-in parameter $\alpha_{e} V_{\text {pull-in }}^{2}$ and its corresponding nanobeam deflection in the presence of the intermolecular forces. The method of identifying the pull-in threshold value is to iterate on the forcing parameter $\alpha_{e} V_{D C}^{2}$ with a reasonable step size and check its value at which the feasible and real solution of the polynomial of $k_{1}$ becomes imaginary and the system becomes unstable, hence undergoing the pull-in structural instability.

First, the electric fringing field and intermolecular force effects are neglected $\left(\alpha_{f f}=\alpha_{v d W}=\alpha_{c a s}=0\right)$ in Equations (14) and (16), and the resulting pull-in parameter $\left(\alpha_{e} V_{p u l l-i n}^{2}\right)$ values are compared with the results of [30]. Both Equations (14) and (16) provide a pull-in parameter of $\approx 66.58$. In [30], this parameter value was numerically calculated to be equal to 70 , and the comparison is satisfactory.

Next, we propose to investigate, as done before, two distinct cases for getting the closed-form expressions of the pull-in parameter under the influence of the van der Waals and Casimir forces, respectively.

\subsubsection{First Case: Van der Waals Force Only $\left(F_{v d W} \neq 0\right.$ and $\left.F_{\text {Casimir }}=0\right)$}

\section{$\sqrt{ }$ Case with the fringing-field effect and without the mid-plane stretching effect:}

First, the mid-plane stretching is neglected and the electric fringing field effect is incorporated in the below closed-form analytical expression. The procedure can be summarized as follows: assuming a different range of values of the van der Waals parameter $\left(\alpha_{v d W}\right)$ ranging from 0-50 along with the electric fringing field parameter $\left(\alpha_{f f}\right)$ ranging from 0-1, Equation (14) numerically and iteratively solved the physical and real solution $k_{1}$, which becomes imaginary. Then, and for each corresponding $\alpha_{v d \mathrm{~W}}$ and $\alpha_{f f}$ value, the corresponding threshold pull-in parameter value of $\left(\alpha_{e} V_{p u l l-i n}^{2}\right)$ is prescribed. Accordingly, a 3D table is constructed correlating the values of the pull-in, van der Waals and electric fringing field parameters all together. The table is first suitably curve fitted with a third order polynomial using the MATLAB curve fitting toolbox as shown in Equation (19), with a calculated RMS error of 0.03, and the outcome equation is then platted as shown in Figure 2.

$$
\begin{aligned}
\alpha_{e} V_{p u l l-i n}^{2} & =66.51-28.95 \alpha_{f f}-1.45 \alpha_{v d W}+9.84 \alpha_{f f}^{2}+0.55 \alpha_{f f} \alpha_{v d W}+2.47 \times 10^{-3} \alpha_{v d W}^{2}+ \\
& -1.464 \alpha_{f f}^{3}-0.145 \alpha_{f f}^{2} \alpha_{v d W}+3 \times 10^{-4} \alpha_{f f} \alpha_{v d W}^{2}-1.3 \times 10^{-5} \alpha_{v d W}^{3}
\end{aligned}
$$

As the pull-in parameter in Equation (23) is a function of both $\alpha_{v d W}$ and $\alpha_{f f}$, the 3D plot shown in Figure 2 is essentially a nonlinear plane. As expected, with the increase in both the gap to width ratio, i.e., the electric fringing field parameter $\left(\alpha_{f f}\right)$ in addition to the van der Waals parameter, the pull-in parameter reduces drastically. 


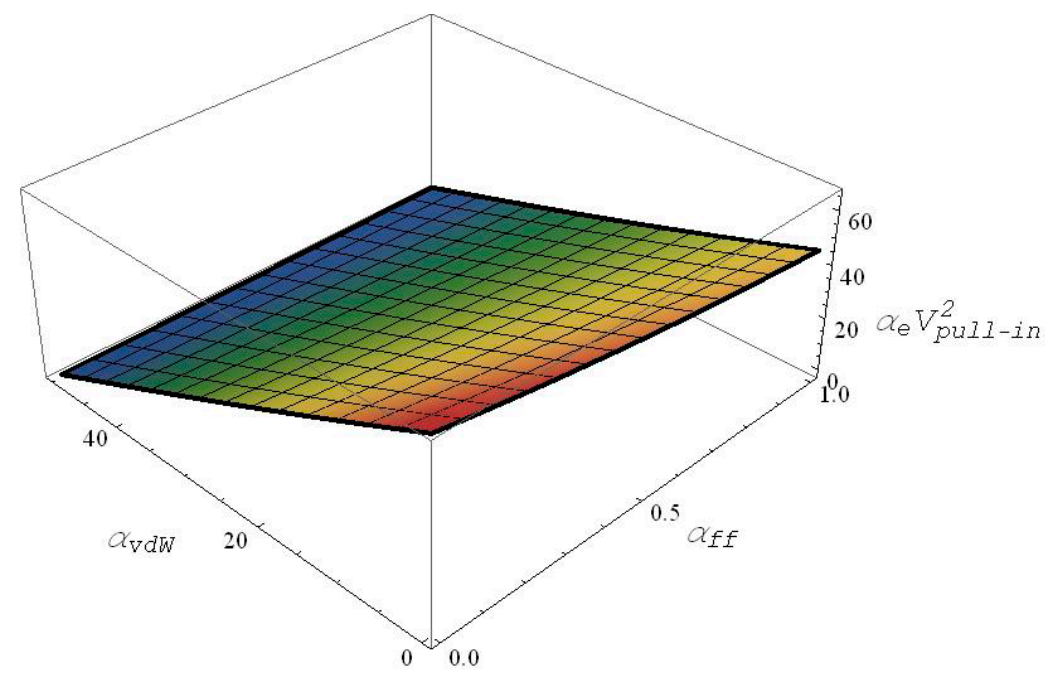

Figure 2. Three-dimensional (3D) plot of the variation of the pull-in parameter with the van der Waals and electric fringing-field parameters.

$\sqrt{ }$ Case with the mid-plane stretching effect and without the fringing-field effect:

Next, the fringing-field effect is neglected, whereas the mid-plane stretching effect is varied in the presence of the van der Waals effect. Similarly as was done in the previous sub-section, a 3D table is constructed correlating the values of the pull-in, van der Waals and mid-plane stretching parameters all together. The table is then curve fitted with a third order polynomial using the MATLAB curve fitting toolbox as shown in Equation (20), with a calculated RMS error of 0.02, and the outcome equation is then platted as shown in Figure 3.

$$
\begin{aligned}
\alpha_{e} V_{p u l l-i n}^{2} & =66.67+0.893 \alpha_{s t}-1.45 \alpha_{v d W}^{2}-3.4 \times 10^{-3} \alpha_{s t}^{2}-5 \times 10^{-3} \alpha_{s t} \alpha_{v d W}+1.9 \times 10^{-3} \alpha_{v d W}^{2}+ \\
& -4.43 \times 10^{-5} \alpha_{s t}^{3}-7.01 \times 10^{-5} \alpha_{s t}^{2} \alpha_{v d W}-2.44 \times 10^{-5} \alpha_{s t} \alpha_{v d W}^{2}-3 \times 10^{-6} \alpha_{v d W}^{3}
\end{aligned}
$$

As the pull-in parameter in Equation (23) is a function of both $\alpha_{v d W}$ and $\alpha_{s t}$, the 3D plot shown in Figure 3 is basically a nonlinear plane. It can also be seen from the figure that an increase in the van der Waals parameter decreases the pull-in values; however, an increase in the mid-plane stretching parameter increases the pull-in values considerably.

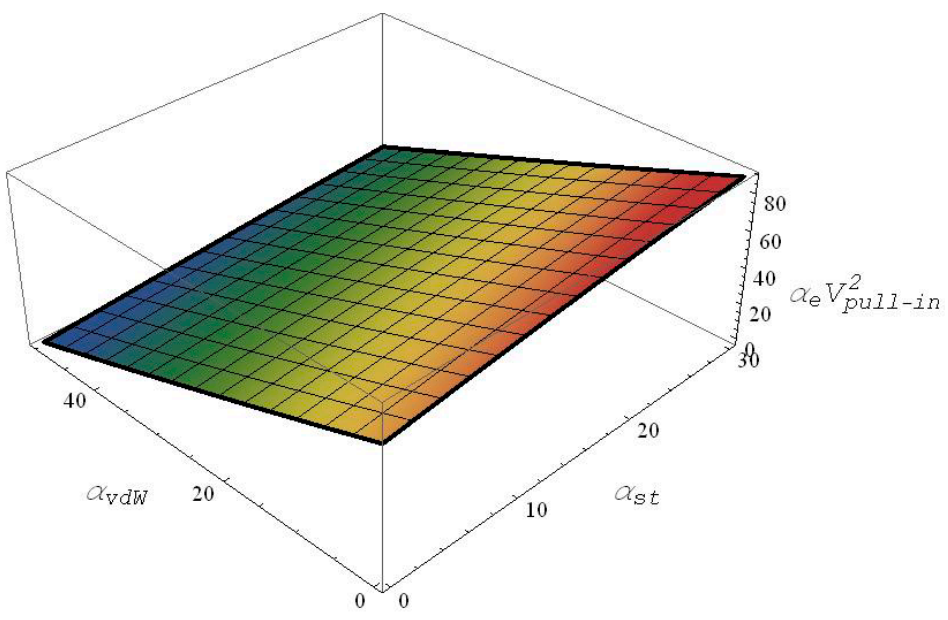

Figure 3. 3D plot of the variation of the pull-in parameter with the van der Waals and mid-plane stretching parameters. 


\subsubsection{Second Case: Casimir Force Only $\left(F_{v d W}=0\right.$ and $\left.F_{\text {Casimir }} \neq 0\right)$}

Here, the pull-in parameter analytical expressions are derived considering the effect of Casimir forces only.

$\sqrt{ }$ Case with the fringing-field effect and without the mid-plane stretching effect:

Next, the fringing-field effect is varied from $0-1$, whereas the mid-plane stretching effect is neglected in the presence of the Casimir force effect. Similarly as was done in the previous sub-sections on the van der Waal force case, a 3D table is constructed correlating values of the pull-in, Casimir and the fringing electric field parameters all together. The table is then curve fitted with a third order polynomial using the MATLAB curve fitting toolbox as shown in Equation (21), with a calculated RMS error of 0.039, and the outcome equation is then platted as shown in Figure 4.

$$
\begin{gathered}
\alpha_{e} V_{p u l l-i n}^{2}=64.76-28.88 \alpha_{f f}-2.03 \alpha_{c a s}+9.97 \alpha_{f f}^{2}+0.742 \alpha_{f f} \alpha_{c a s}+0.0126 \alpha_{c a s}^{2}+ \\
-1.52 \alpha_{f f}^{3}-0.189 \alpha_{f f}^{2} \alpha_{c a s}+4 \times 10^{-4} \alpha_{f f} \alpha_{c a s}^{2}-1 \times 10^{-4} \alpha_{c a s}^{3}
\end{gathered}
$$

As seen from Figure 4, and as expected, the increase in both the gap to width ratio (i.e., the fringing field parameter), as well as the Casimir parameter both cause a significant decrease of the pull-in parameter.

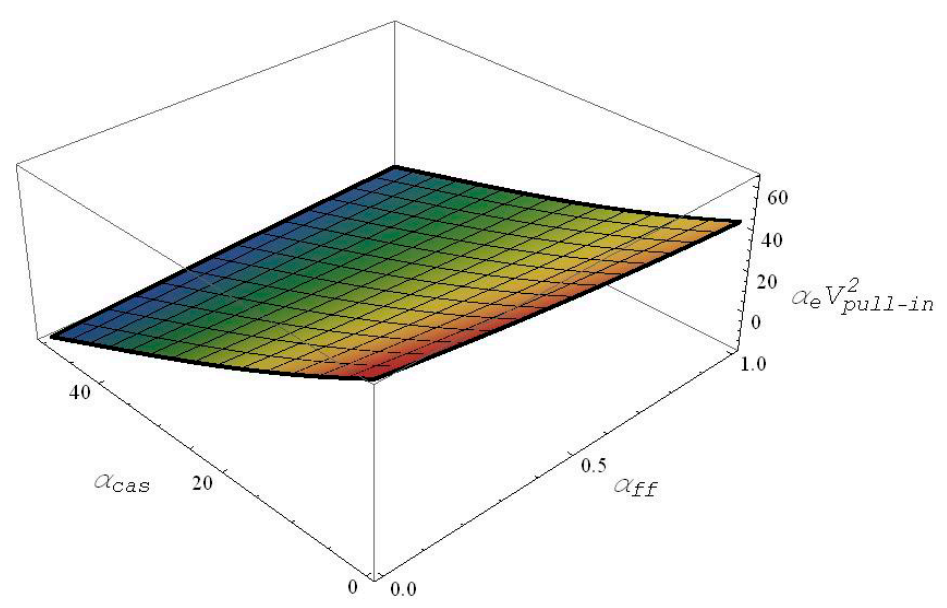

Figure 4. $3 \mathrm{D}$ plot of the variation of the pull-in parameter with the Casimir and electric fringing-field parameters.

$\sqrt{ }$ Case with the mid-plane stretching effect and without the fringing-field effect:

Next, the electric fringing effect is neglected, and the mid-plane stretching effect is investigated in the presence of Casimir effect. Similarly as was done in the previous sub-section, a 3D table is constructed correlating the values of the pull-in, van der Waals and mid-plane stretching parameters all together. The table is then curve fitted with a third order polynomial using the MATLAB curve fitting toolbox as shown in Equation (22), with a calculated RMS error of 0.03, and the outcome equation is then platted as shown in Figure 5.

$$
\begin{aligned}
\alpha_{e} V_{\text {pull-in }}^{2} & =64.87+0.64 \alpha_{\text {st }}-2 \alpha_{\text {cas }}-1 \times 10^{-3} \alpha_{s t}^{2}-5.2 \times 10^{-3} \alpha_{\text {st }} \alpha_{\text {cas }}+9.4 \times 10^{-3} \alpha_{c a s}^{2}+ \\
& -1.9 \times 10^{-5} \alpha_{s t}^{3}+8.3 \times 10^{-5} \alpha_{s t}^{2} \alpha_{\text {cas }}-9.3 \times 10^{-5} \alpha_{s t} \alpha_{c a s}^{2}-3.6 \times 10^{-6} \alpha_{\text {cas }}^{3} ;
\end{aligned}
$$

It is apparent from Figure 5 that any assumed increase of the Casimir parameter decreases the pull-in parameters, whereas any assumed increase in the mid-plane stretching effect increases the pull-in values considerably. 


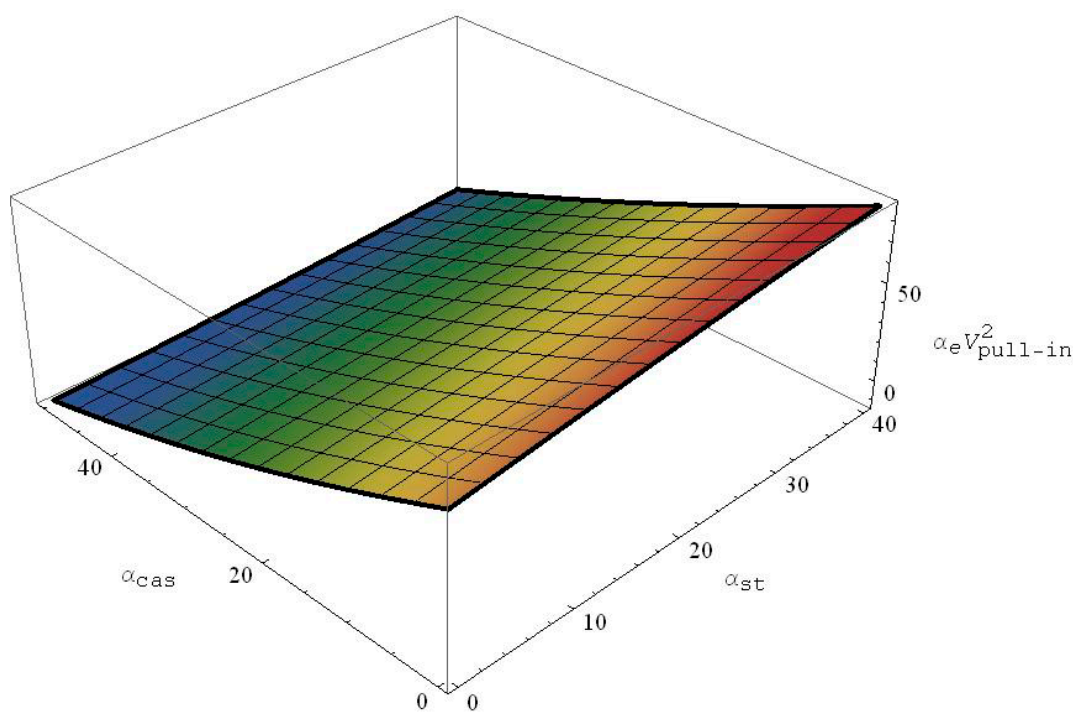

Figure 5. 3D plot of the variation of the pull-in parameter with the Casimir and mid-plane stretching parameters.

\subsection{Comparison with the Literature of the Pull-In Parameter in Presence of Van der Waals and Casimir Forces}

In this last sub-section of the results analysis, all the above computed analytical expressions of the pull-in parameters will be compared with other previously published results for validation purposes. Tables 5-8 summarize the comparison for both cases while assuming a non-zero van der Waals parameter and also when assuming a non-zero Casimir parameter.

Table 5 displays a quantitative comparison of the calculated pull-in parameters using Equation (21) respectively with the reported results of [14]. In the table, various Casimir parameters $\alpha_{c a s}$ are selected, for two different fringing field parameters (i.e., $\alpha_{f f}=0$ and 1 ), and neglecting mid-plane stretching $\left(\alpha_{s t}=0\right)$. The table shows that the pull-in parameter values obtained analytically are slightly lower than the numerical value of [14]. This small discrepancy could be attributed to the numerical approximations used in [14] and to the negligence of the higher-order modes in the present work.

Table 5. Variation of the pull-in parameter with the Casimir parameter as obtained using Equation (21) and then as compared with the results of [14].

\begin{tabular}{ccccc}
\hline \multirow{2}{*}{$\alpha_{\text {cas }}$} & \multicolumn{2}{c}{$\alpha_{f f}=\alpha_{\text {st }}=\mathbf{0}$} & \multicolumn{2}{c}{$\alpha_{f f}=\mathbf{1} ; \alpha_{\text {st }}=\mathbf{0}$} \\
\cline { 2 - 5 } & Equation (21) & Results of [14] & Equation (21) & Results of [14] \\
\hline 10 & 48.57 & 49.43 & 32.63 & 33.81 \\
20 & 30.38 & 31.08 & 19.78 & 20.77 \\
30 & 13.37 & 14.07 & 8.18 & 8.91 \\
\hline
\end{tabular}

Next, the same pull-in parameters will be computed while the mid-plane stretching is assumed to be non-zero, neglecting the fringing field effect, and considering both the influences of the van der Waals and of the Casimir forces effects. The results are then compared with the outcomes [10] and summarized in Tables 6 and 7, respectively. It can again be noticed from both tables that the present analytical approach slightly underestimates the pull-in voltage parameters as compared to the published values in [11], which can be over attributed to the one-mode approximation we assumed in out analytical/numerical approach.

Finally, Table 8 delivers a quantitative comparison of pull-in voltage (in Volts) with and without including the mid-plane stretching effect and then compared to the results of [12]. The comparison was performed assuming a doubly-clamped nanobeam with an initial gap size of $d=20 \mathrm{~nm}$, a thickness 
$h=3.5 \mathrm{~nm}$, a width of $b=18 \mathrm{~nm}$, a Young's modulus of elasticity of $E=166 \mathrm{GPa}$ and three assumed beam lengths of $L=130 \mathrm{~nm}, L=150 \mathrm{~nm}$ and $L=180 \mathrm{~nm}$. Table 8 first shows that the comparison with the results of [12] has an acceptable agreement. Second, it is noticed from the same comparison table that neglecting the mid-plane stretching effect will lead to a significant difference in the pull-in voltage estimation. It is also evident from the same table that the pull-in voltages obtained through our proposed approach while incorporating both effects of the electric fringing field and the mid-plane stretching agree well in both the van der Waals and Casimir force cases. For the case of Casimir force only, the comparison is even more in agreement as compared with the outcomes when considering only van der Waals force. This implies that for given beam dimensions and a 20-nm initial gap size, the intermolecular effects can be properly modeled by considering the Casimir attraction force rather than the van der Waals force. Therefore, this last assumption can yet serve as quick design guidelines in the NEMS community.

Table 6. Variation of the pull-in parameter with the van der Waals parameter as obtained using Equation (20) and then as compared with the results of [11].

\begin{tabular}{ccc}
\hline \multirow{2}{*}{$\alpha_{v d W}$} & \multicolumn{2}{c}{$\alpha_{\text {st }}=\mathbf{6}$} \\
\cline { 2 - 3 } & Equation (20) & Results of [11] \\
\hline 10 & 62.57 & 63.21 \\
20 & 46.18 & 47.9 \\
30 & 29.1 & 33.2 \\
\hline
\end{tabular}

Table 7. Variation of the pull-in parameter with the Casimir parameter as obtained using Equation (22) and then as compared with the results of [11].

\begin{tabular}{ccc}
\hline \multirow{2}{*}{$\boldsymbol{\alpha}_{\text {cas }}$} & \multicolumn{2}{c}{$\boldsymbol{\alpha}_{\text {st }}=\mathbf{6}$} \\
\cline { 2 - 3 } & Equation (22) & Results of [11] \\
\hline 10 & 54.24 & 55.8 \\
20 & 34.31 & 35.77 \\
30 & 14.68 & 18.2 \\
\hline
\end{tabular}

Table 8. Variation of the pull-in voltage (in Volts) with both the van der Waals and Casimir parameters as obtained using Equations (20) and (22), respectively, and then as compared with the results of [12].

\begin{tabular}{ccccccc}
\hline \multirow{2}{*}{$\boldsymbol{L}(\mathbf{n m})$} & \multicolumn{3}{c}{ Van der Waals Force Case } & \multicolumn{3}{c}{ Casimir Force Case } \\
\cline { 2 - 7 } & $\begin{array}{c}\text { Equation (20) } \\
\boldsymbol{\alpha}_{\boldsymbol{s t}}=\mathbf{0}\end{array}$ & $\begin{array}{c}\text { Equation (20) } \\
\boldsymbol{\alpha}_{\text {st }} \neq \mathbf{0}\end{array}$ & $\begin{array}{c}\text { Results of [12] } \\
\boldsymbol{\alpha}_{\text {st }} \neq \mathbf{0}\end{array}$ & $\begin{array}{c}\text { Equation (22) } \\
\boldsymbol{\alpha}_{\text {st }}=\mathbf{0}\end{array}$ & $\begin{array}{c}\text { Equation (22) } \\
\boldsymbol{\alpha}_{\text {st }} \neq \mathbf{0}\end{array}$ & $\begin{array}{c}\text { Results of [12] } \\
\boldsymbol{\alpha}_{\text {st }} \neq \mathbf{0}\end{array}$ \\
\hline 130 & 12.91 & 15.76 & 14.41 & 12.64 & 14.45 & 14.37 \\
150 & 9.69 & 11.83 & 10.82 & 9.47 & 10.91 & 10.77 \\
180 & 6.72 & 8.28 & 7.51 & 6.53 & 7.54 & 7.43 \\
\hline
\end{tabular}

\section{Conclusions}

The present work proposes simple and yet generalized analytical expressions for determining doubly-clamped electrically-actuated nanobeam-based NEMS actuator design limits for various critical design parameters along with the appropriate static pull-in parameters under the influence of the mid-plane stretching, the electric fringing field, the van der Waals and the Casimir forces. Firstly, the coupled electromechanical problem of the nano-actuator is proposed and discretized using the so-called Galerkin modal discretization technique. In the subsequent steps, the resulting integrals and the higher order polynomials are solved numerically to get closed-form expressions for the maximum nanobeam detachment length, its respective minimum gap size and the pull-in parameter expressions when considering only the first mode of the nanobeam deflection. It has been further 
observed through extensive examinations that the incorporation of the intermolecular forces and the electric fringing field effect result in a significant decrease in the pull-in voltage estimation, whereas the presence of the mid-plane stretching parameter shows contrary effects. Finally, the derived expressions and results were all compared and validated quantitatively with other reported literature showing reasonable agreement.

The main advantage of the proposed approach in this work is that the derived closed-form analytical expressions do not include any complicated terms or complex mathematical operations unlike in the reported literature; hence, it can be willingly used by NEMS designers for quick and closer estimation of critical design parameters to avoid any unprecedented structural damage during fabrication or operation. The derived analytical expressions were presented to produce fair outcomes for most common design parameters of nanobeam-based actuators. For nanobeams with a higher stretching effect, it was found that these expressions yield less exact approximations due to the limitations of the single-mode approximation. In such cases, a multi-mode-based numerical model is to be implemented for more accurate results.

Finally, it is worth mentioning that this investigation treated both intermolecular forces independently. Nonetheless, the obtained analytical expression can be further coupled to account for the joint effect of both attractive forces. Consequently, the results could be further improved and would be more comprehensive for scenarios where the two forces are of comparable strength.

Author Contributions: Hassen M. Ouakad suggested the model, performed the equations derivation, derived the reduced-order model, simulated the numerical results and drafted the manuscript. Jihad E. AlQasimi assisted in the reduced-order modeling and in the manuscript preparation.

Conflicts of Interest: The authors declare no conflict of interest.

\section{References}

1. Edward, L.S. MEMS and NEMS: Systems, Devices, and Structures; CRC Press: Boca Raton, FL, USA, 2002.

2. Arani, A.G.; Ghaffari, M.; Jalilvand, A.; Kolahchi, R. Nonlinear nonlocal pull-in instability of boron nitride nanoswitches. Acta Mech. 2013, 224, 3005-3019. [CrossRef]

3. Hajati, A.; Kim, S.G. Ultra-wide bandwidth piezoelectric energy harvesting. Appl. Phys. Lett. 2011, $99,83105$. [CrossRef]

4. Zhang, W.-M.; Yan, H.; Peng, Z.-K.; Meng, G. Electrostatic pull-in instability in MEMS/NEMS: A review. Sens. Actuators A 2014, 214, 187-218. [CrossRef]

5. Soroush, R.; Koochi, A.; Kazemi, A.S.; Noghrehabadi, A.; Haddadpour, H.; Abadyan, M. Investigating the effect of Casimir and van derWaals attractions on the electrostatic pull-in instability of nano-actuators. Phys. Scr. 2010, 82, 45801. [CrossRef]

6. Ramezani, A.; Alasty, A.; Akbari, J. Analytical investigation and numerical verification of Casimir effect on electrostatic nano-cantilevers. Microsyst. Technol. 2008, 14, 145-157. [CrossRef]

7. Ramezani, A.; Alasty, A.; Akbari, J. Closed-form solutions of the pull-in instability in nano-cantilevers under electrostatic and intermolecular surface forces. Int. J. Solids Struct. 2007, 44, 4925-4941. [CrossRef]

8. Farrokhabadi, A.; Abadian, N.; Rach, R.; Abadyan, M. Theoretical modeling of the Casimir forceinduced instability in freestanding nanowires with circular cross-section. Phys. E 2014, 63, 67-80. [CrossRef]

9. Ramezani, A.; Alasty, A.; Akbari, J. Closed-form approximation and numerical validation of the influence of van der Waals force on electrostatic cantilevers at nano-scale separations. Nanotechnology 2008, 19, 15501. [CrossRef] [PubMed]

10. Zand, M.M.; Ahmadian, M.T. Dynamic pull-in instability of electrostatically actuated beams incorporating Casimir and van derWaals forces. Proc. Inst. Mech. Eng. Part C 2010, 224, 2037-2046. [CrossRef]

11. Lin, W.-H.; Zhao, Y.-P. Dynamic behaviour of nanoscale electrostatic actuators. Chin. Phys. Lett. 2003, 20, 2070-2073.

12. Yang, J.; Jia, X.L.; Kitipornchai, S. Pull-in instability of nano-switches using nonlocal elasticity theory. J. Phys. D Appl. Phys. 2008, 41, 35103. [CrossRef]

13. Beni, Y.T.; Karimipour, I.; Abadyan, M. Modeling the instability of electrostatic nano-bridges and nano-cantilevers using modified strain gradient theory. Appl. Math. Model. 2015, 39, 2633-2648. [CrossRef] 
14. Koochi, A.; Kazemi, A.S.; Beni, Y.T.; Yekrangi, A.; Abadyan, M. Theoretical study of the effect of Casimir attraction on the pull-in behavior of beam-type NEMS using modified Adomian method. Phys. E 2010, 43, 625-632. [CrossRef]

15. Taghavi, N.; Nahvi, H. Pull-in instability of cantilever and fixed-fixed nano-switches. Eur. J. Mech. A/Solids 2013, 41, 123-133. [CrossRef]

16. Shokravi, M. Dynamic pull-in and pull-out analysis of viscoelastic nanoplates under electrostatic and Casimir forces via sinusoidal shear deformation theory. Microelectron. Reliab. 2017, 71, 17-28. [CrossRef]

17. Wang, Z.-Q.; Zhao, Y.-P.; Huang, Z.-P. The effects of surface tension on the elastic properties of nano structures. Int. J. Eng. Sci. 2010, 48, 140-150. [CrossRef]

18. Jin, H.; Lilley, C.M. Surface stress effect on bending resonance of nanowires with different boundary conditions. Appl. Phys. Lett. 2008, 93, 263108. [CrossRef]

19. Haifei, Z.; Gu, Y.; Park, H.S. Beat phenomena in metal nanowires, and their implications for resonance-based elastic property measurements. Nanoscale 2012, 4, 6779-6785.

20. Sedighi, H.M.; Ouakad, H.M.; Khooran, M. Instability characteristics of free-standing nanowires based on the strain gradient theory with the consideration of casimir attraction and surface effects. Metrol. Meas. Syst. 2017, 24, 489-507. [CrossRef]

21. Lifshitz, E.M. The theory of molecular attractive forces between solids. J. Exp. Theor. Phys. 1956, 29, 73-83.

22. Dykman, M. Fluctuating Nonlinear Oscillators: From Nanomechanics to Quantum Superconducting Circuits; Oxford University Press: Oxford, UK, 2012.

23. Ouakad, H.M. Nonlinear structural mechanics of micro-and nanosystems. In Nonlinearity in Energy Harvesting Systems; Springer International Publishing: Cham, Switzerland, 2016; pp. 127-195.

24. Elmer, H.S.; Senturia, S.D. Generating efficient dynamical models for microelectromechanical systems from a few finite-element simulation runs. J. Microelectromech. Syst. 1999, 8, 280-289.

25. Najar, F.; Choura, S.; Abdel-Rahman, E.M.; El-Borgi, S.; Nayfeh, A.H. Dynamic analysis of variable-geometry electrostatic microactuators. J. Micromech. Microeng. 2006, 16, 2449. [CrossRef]

26. Nayfeh, A.H.; Younis, M.I.; Abdel-Rahman, E.M. Characterization of the mechanical behavior of an electrically actuated microbeam. J. Micromech. Microeng. 2002, 12, 759-763.

27. Ouakad, H.M.; Younis, M.I. Modeling and simulations of collapse instabilities of microbeams due to capillary forces. Math. Probl. Eng. 2009, 2009, 1-16. [CrossRef]

28. Ouakad, H.M.; Sedighi, H.M. Rippling effect on the structural response of electrostatically actuated single-walled carbon nanotube based NEMS actuators. Int. J. Non-Linear Mech. 2016, 87, 97-108. [CrossRef]

29. Ouakad, H.M. Comprehensive numerical modeling of the nonlinear structural behavior of MEMS/NEMS electrostatic actuators under the effect of the van der Waals forces. Microsyst. Technol. 2017, 23, 5903-5910. [CrossRef]

30. Younis, M.I. MEMS Linear and Nonlinear Statics and Dynamics; Springer Science \& Business Media: New York, NY, USA, 2011; Volume 20.

(C) 2018 by the authors. Licensee MDPI, Basel, Switzerland. This article is an open access article distributed under the terms and conditions of the Creative Commons Attribution (CC BY) license (http://creativecommons.org/licenses/by/4.0/). 